\title{
Valuative characterization of central extensions of algebraic tori on Krull domains *
}

\author{
Haruhisa NAKAJIMA ${ }^{\dagger}$ \\ Department of Mathematics, J. F. OBerLin University \\ Tokiwa-machi, MACHIDA, TOKYO 194-0294, JAPAN
}

\begin{abstract}
Let $G$ be an affine algebraic group with an algebraic torus $G^{0}$ over an algebraically closed field $K$ of an arbitrary characteristic $p$. We show a criterion for $G$ to be a finite central extension of $G^{0}$ in terms of invariant theory of all regular actions of any closed subgroup $H$ containing $Z_{G}\left(G^{0}\right)$ on affine Krull $K$-schemes such that invariant rational functions are locally fractions of invariant regular functions. Consider an affine Krull $H$-scheme $X=\operatorname{Spec}(R)$ and a prime ideal $\mathfrak{P}$ of $R$ with ht $(\mathfrak{P})=\operatorname{ht}\left(\mathfrak{P} \cap R^{H}\right)=1$. Let $\mathcal{I}(\mathfrak{P})$ denote the inertia group of $\mathfrak{P}$ under the action of $H$. The group $G$ is central over $G^{0}$ if and only if the fraction $\mathrm{e}\left(\mathfrak{P}, \mathfrak{P} \cap R^{H}\right) / \mathrm{e}\left(\mathfrak{P}, \mathfrak{P} \cap R^{\mathcal{I}(\mathfrak{P})}\right)$ of ramification indices is equal to $1(p=0)$ or to the $p$-part of the order of the group of weights of $G^{0}$ on $R^{\mathcal{I}(\mathfrak{P}))}$ vanishing on $R^{\mathcal{I}(\mathfrak{P}))} / \mathfrak{P} \cap R^{\mathcal{I}(\mathfrak{P})}(p>0)$ for an arbitrary $X$ and $\mathfrak{P}$.
\end{abstract}

MSC: primary 13A50, 14R20, 20G05; secondary 14L30, 14M25

Keywords: Krull domain; ramification index; algebraic group; algebraic torus; character group; invariant theory

\section{Introduction}

1.A. We consider affine algebraic groups and affine schemes over a fixed algebraically closed field $K$ of an arbitrary characteristic $p$. For an affine group $G$, denote by $G^{0}$ its identity component. Let $\rho: G \rightarrow G L(V(\rho))$ be a finite dimensional rational representation of $G$ and $A=K[V(\rho)]$ a $K$-algebra of polynomial functions on $V(\rho)$. We have the inclusions

$$
A^{G} \subseteq A^{G^{0}} \subseteq A^{\left(G^{0}\right)^{\prime}} \subseteq A^{\Re a d_{u}\left(G^{0}\right)}
$$

of rings of invariants, where $\mathfrak{R} a d_{u}\left(G^{0}\right)$ is the unipotent radical of $G^{0}$ and $\left(G^{0}\right)^{\prime}$ is the inverse image in $G^{0}$ of the semi-simple part of $G^{0} / \mathfrak{R} a d_{u}\left(G^{0}\right)$. These rings are Krull domains but, they are not necessarily finitely generated over $K$ if $\mathfrak{R} a d_{u}\left(G^{0}\right)$ is nontrivial. Since $A^{G}$ is obtained as a ring of invariants of the Krull $K$-domain $A^{\left(G^{0}\right)^{\prime}}$ (resp. $\left.A^{\mathfrak{R}\left(G^{0}\right)}\right)$ under the action of $G /\left(G^{0}\right)^{\prime}$ (resp. $\left.G / \mathfrak{R} a d_{u}\left(G^{0}\right)\right)$ whose identity component is an algebraic torus (resp. a reductive group). On the other hand by slice étale theorem (e.g., 13]), it needs to study on invariants of the stabilizer $G_{P}$ of a suitable point $P$, which may not be connected. Moreover reductivity of algebraic groups is characterized by pseudo-reflections of actions on Krull $K$-domains (cf. [12). Consequently in order to examine $A^{G}$ we have to study rings of invariants of Krull $K$-domains with the above group actions in general settings.

On (1.1), in the case where $G^{0}$ is semisimple and $p=0$, if $A^{G}$ is polynomial ring then $A^{G^{0}}$ is a complete intersection (cf. [14]). This seems to be useful to classify such

\footnotetext{
*This is a version on August 3, 2016.

${ }^{\dagger}$ Partially supported by Grant No. 26400019: the Japan Society for the Promotion of Sciences.
} 
representations of $G$. The author studied in 9 the representation $\rho$ of $G$ with an algebraic torus $G^{0}$ such that $R^{G}$ is a polynomial ring in the case of $p=0$. Our result suggests there are differences in invariant theory of finite central extensions and finite non-central extensions of a torus related to the role of pseudo-reflections. Hence we will clarify the background of this phenomenon in this paper and advance Problem 1.1 as follows.

1.B. Affine $K$-schemes $X$ are affine schemes of commutative $K$-algebras $\mathcal{O}(X)=R$ of global sections which are not necessarily finite generated as algebras over $K$. Let $\mathcal{Q}(R)$ denote the total quotient ring of $R$. We say an action $(X, G)$ of an affine algebraic group $G$ on an affine $K$-scheme $X$ is regular, when $G$ acts rationally on the $K$-algebra $R$ as $K$-algebra automorphisms, i.e., $R$ is regarded as a rational $G$-module. In this case we denote by $X / / G$ the affine $K$-scheme defined by $R^{G}$ which is the $K$-subalgebra consisting of all invariants of $R$ under the action of $G$ and by

$$
\pi_{X, G}: X \rightarrow X / / G
$$

the morphism induced from the inclusion $R^{G} \hookrightarrow R$. Furthermore $(X, G)$ is said to be effective if $\operatorname{Ker}(\mathrm{G} \rightarrow$ Aut $\mathrm{X})$ is finite.

1.C. The affine $K$-scheme $X=\operatorname{Spec}(R)$ is said to be Krull, if $R$ is a Krull $K$ domain. In this case let $\mathrm{e}\left(\mathfrak{P}, \mathfrak{P} \cap R^{G}\right)$ be the ramification index is defined to satisfy $\left(\mathfrak{P} R_{\mathfrak{P}}\right)^{\mathrm{e}\left(\mathfrak{P}, \mathfrak{P} \cap R^{G}\right)}=\left(\mathfrak{P} \cap R^{G}\right) R_{\mathfrak{P}}$ for a prime ideal $\mathfrak{P}$ of $R$ of $\operatorname{ht}(\mathfrak{P})=\operatorname{ht}\left(\mathfrak{P} \cap R^{G}\right)=1$. The ramification indices are related to the order of pseudo-reflections of $G$ which are important in the study on relative invariants (cf. [16, 8, 11]).

Problem 1.1 (Reduction of ramification indices to finite subgroup quotients) Is there a finite subgroup $I_{\mathfrak{P}}$ of $G$ such that $\mathrm{e}\left(\mathfrak{P}, \mathfrak{P} \cap R^{G}\right)$ is equal to a product of $\mathrm{e}\left(\mathfrak{P}, \mathfrak{P} \cap R^{I_{\mathfrak{P}}}\right)$ and $p^{n}$ for some computable $n \geqq 0$ ?

This is affirmative in the restricted case treated in [9] and plays a key role in the classification of representations of disconnected tori with regular rings of invariants. Now put

$$
\mathcal{I}_{G}(\mathfrak{P}):=\{\sigma \in G \mid \sigma(x)-x \in \mathfrak{P}(\forall x \in R)\} .
$$

This group is finite on $X$ for $G$ with a reductive $G^{0}$ (cf. [12]). In this paper we solve Problem 1.1 completely for $G$ with an algebraic torus $G^{0}$. It should be noted that this problem is not affirmative, if $G^{0}$ is not a torus (cf. Example 6.2).

1.D. The present paper is a continuation of $[10$ and completes its studies. We will establish a characterization of a finite central extension of a torus in terms of ramification indices in invariant theory. In order to study on them, it is natural to treat regular actions on Krull $K$-domains. Invariants of finite central extensions of algebraic tori with relations to pseudo-reflections (or ramification theory) are useful in studying the obstruction groups for cofreeness defined in 11. Those groups evaluate failure of the Russian conjecture for actions of algebraic tori on normal varieties (cf. Sect.1 and Examples 5.6, 5.7 of [11]) in characteristic zero.

1.F. Let $\mathfrak{X}(G)$ be the group of rational characters of $G$ expressed as an additive group with zero denoted to $0_{G}$. For a rational $G$-module $M$, put

$$
\left\{\begin{array}{l}
\mathfrak{X}(G)^{M}:=\left\{\chi \in \mathfrak{X}(G) \mid M_{\chi} \neq\{0\}\right\} \\
\mathfrak{X}(G)_{M}:=\left\{\chi \in \mathfrak{X}(G)^{M} \mid M_{-\chi} \neq\{0\}\right\}
\end{array} .\right.
$$

Here $M_{\chi}$ stands for the space $\{x \in M \mid \sigma(x)=\chi(\sigma) x\}$ of relative invariants of $G$ in $M$ relative to $\chi$. Clearly $\mathfrak{X}(G)_{M}$ is a subgroup of $\mathfrak{X}(G)$, which shall be used in Sect. 3 . 
1.G. Let us explain our main result as follows. Suppose that $G^{0}$ is an algebraic torus and let $H$ be a closed subgroup of $G$ containing the centralizer $Z_{G}\left(G^{0}\right)$ of $G^{0}$ in $G$. Consider a regular action $(X, H)$ of $H$ on an affine Krull $K$-scheme $X=\operatorname{Spec}(R)$. For a prime ideal $\mathfrak{P}$ of $R$ such that ht $(\mathfrak{P})=\operatorname{ht}\left(\mathfrak{P} \cap R^{H}\right)=1$, we define

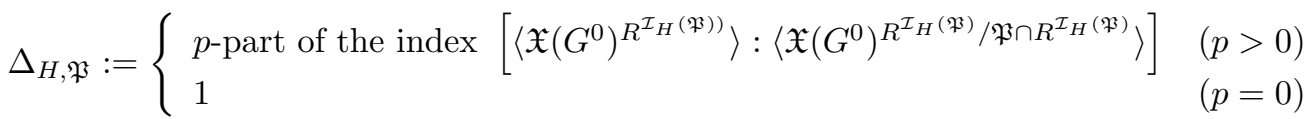

The main theorem of this paper is as follows:

Theorem 1.2 Suppose that $G^{0}$ is an algebraic torus. Then the following conditions are equivalent:

(i) $G=Z_{G}\left(G^{0}\right)$

(ii) For an arbitrary closed subgroup $H$ of $G$ containing $Z_{G}\left(G^{0}\right)$, the following conditions hold for any effective regular action $(X, H)$ on an arbitrary affine Krull $K$-scheme $X=\operatorname{Spec}(R)$ such that $\mathcal{Q}\left(R^{G^{0}}\right)=\mathcal{Q}(R)^{G^{0}}$ : If $\mathfrak{P}$ is any prime ideal of $R$ with $\operatorname{ht}(\mathfrak{P})=\operatorname{ht}\left(\mathfrak{P} \cap R^{H}\right)=1$, the following equality holds:

$$
\mathrm{e}\left(\mathfrak{P}, \mathfrak{P} \cap R^{H}\right)=\mathrm{e}\left(\mathfrak{P}, \mathfrak{P} \cap R^{\mathcal{I}_{H}(\mathfrak{P})}\right) \cdot \Delta_{H, \mathfrak{P}}
$$

It should be noted that the right hand side of the equalities in (ii) are obtained from datum on finite group quotients, because $\mathcal{I}_{H}(\mathfrak{P})$ is finite on $X$. The implication (i) $\Rightarrow$ (ii) can be regarded as a generalization of a known result of reduced ramification indices of Krull domains under the actions of finite Galois groups.

1.H. We prepare some results on invariant rational functions in Sect.2 which are helpful for replacing stabilities of algebraic group actions with conditions on quotient fields. Also we prepare the results on rational characters in Sect. 3 which are useful in the calculation of ramification indices. In Sect.4 the structure of inertia groups of toric quotients is studied. In Sect.5 some auxiliary results in Sect.3 of 10 are generalized from the case of normal varieties to one of Krull schemes. The results mentioned in Sect. $1 \sim$ Sect.4 are devoted to the proof the theorem as above which is recognized as a generalization of the main result of [10. Its proof shall be completed in the last section of this paper.

Notations and Comments The following notations are used without explanation:

- Let $p$ denote always the characteristic of an fixed algebraically closed field $K$.

- Let $\mathrm{U}(R)$ (resp. $\mathcal{Q}(R)$ ) denote the unit group (resp. the total quotient ring) of a commutative ring $R$. Let ht $(\mathfrak{I})$ denote the height of an ideal $\mathfrak{I}$ of $R$.

- For a morphism $\gamma: H \rightarrow G$ of groups, let $Z_{G}(H)$ denote the centralizer of a subset $\gamma(H)$ in $G$ (i.e., $\left.Z_{G}(H)=\{\sigma \in G \mid \sigma \cdot \gamma(\tau)=\gamma(\tau) \cdot \sigma(\tau \in H)\}\right)$.

- For a subset $C$ of a group $G$, let $\langle C\rangle$ denote the subgroup of $G$ generated by $C$. For a subgroup $N$ of $G$, let $[G: N]$ denote the index of $N$ in $G$.

- In the case where a group $G$ acts on a set $X$, consider the associated morphism $G \rightarrow \operatorname{Sym}(X)$ from $G$ to the (symmetric) group of all bijective transformations on $X$. Let $\left.\sigma\right|_{X}$ denote the image of $\sigma \in G$ under the canonical morphism $G \rightarrow \operatorname{Sym}(X)$. Moreover let $\left.G\right|_{X}$ be $\operatorname{Im}(G \rightarrow \operatorname{Sym}(X))$. We call $\operatorname{Ker}(G \rightarrow$ Aut $X)$ the ineffective kernel of the action $(X, G)$. If it is trivial (resp. finite), the action of $G$ on $X$ is said to be faithful (resp. effective).

- The $p$-part $n_{[p]}$ and the $p^{\prime}$-part $n_{\left[p^{\prime}\right]}$ of $n \in \boldsymbol{N}$ are the natural numbers in such a way that $n=p^{e} \cdot n_{\left[p^{\prime}\right]}, e \geqq 0, n_{\left[p^{\prime}\right]}$ is not divisible by $p$ and $n_{[p]}=p^{e}$ if $\mathrm{p}$ is a prime number. When $p=0$, we define that the 0 -part $n_{[0]}=1$ and the $0^{\prime}$-part $n_{\left[0^{\prime}\right]}=n$ of $n \in \boldsymbol{N}$. 
- The symbol $\sharp(Y)$ denotes the cardinality of a set $Y$. The symbol $\sharp_{p}(Y)$ (resp. $\sharp_{p^{\prime}}(Y)$ ) denotes the $p$-part (resp. $p^{\prime}$-part ) of $\sharp(Y)$ of a finite set $Y$ if $p$ is prime or 0 .

- For subsets $A, B$ of $C$, let $A \backslash B$ denote the difference set.

\section{Preliminaries}

2.A. For a commutative ring $A$, let $\mathrm{Ht}_{1}(A)$ be the set consisting of prime ideals of $A$ of height 1 . For a prime ideal $\mathfrak{p} \in \mathrm{Ht}_{1}(B)$ of a subring $B$ of $A$, let

$$
\operatorname{Over}_{\mathfrak{p}}(A)=\left\{\mathfrak{P} \in \operatorname{Ht}_{1}(A) \mid \mathfrak{P} \cap B=\mathfrak{p}\right\}
$$

and $\operatorname{Ht}_{1}(A, B)=\left\{\mathfrak{P} \in \mathrm{Ht}_{1}(A) \mid \operatorname{ht}(\mathfrak{P} \cap B)=1\right\}$.

2.B. Let $H$ be a group acting on $A$ as ring automorphisms. For a prime ideal $\mathfrak{Q}$ of $A$, let

$$
\begin{gathered}
\mathcal{D}_{H}(\mathfrak{Q})=\{\sigma \in H \mid \sigma(\mathfrak{Q})=\mathfrak{Q}\}, \\
\mathcal{I}_{H}(\mathfrak{Q})=\operatorname{Ker}\left(\mathcal{D}_{H}(\mathfrak{Q}) \underset{\text { can. }}{\rightarrow} \operatorname{Aut}(A / \mathfrak{Q})\right)
\end{gathered}
$$

which are respectively called the decomposition (reps. inertia) group of $H$ at $\mathfrak{Q}$. Let $A^{H}$ denote the subring of $A$ consisting of invariant elements of $A$ under the action of $H$, whose elements are called invariants of $A$ under $H$. If $A$ is an integrally closed domain, then so is $A^{H}$. For convenience, we note that the Galois theory of integrally closed domains with finite group actions can be found in Chap. $\mathrm{V}$ of [7] and its étale version is given in Exp. $\mathrm{V}$ of 4 with locally noetherian conditions. The ramification theory for Dedekind domains with finite group actions is treated in Chap. V of [17. Clearly $\mathcal{Q}(A)^{H}=\mathcal{Q}\left(A^{H}\right)$ for a finite subgroup $H$ of $\operatorname{Aut} A$ of an integral domain $A$. However the equality $\mathcal{Q}(A)^{H}=\mathcal{Q}\left(A^{H}\right)$ is not true unless $H$ is finite as follows.

Example 2.1 Let $A=K\left[X_{1}, X_{2}, X_{3}, X_{4}\right]$ be a polynomial ring over $K$ with four variables $X_{i}(1 \leq i \leq 4)$. Let $G$ be an algebraic torus $\left(\mathbf{G}_{\mathbf{m}}\right)^{2}$ of rank 2 . Suppose any $\sigma_{s, t}=(s, t) \in \mathbf{G}_{\mathbf{m}} \times \mathbf{G}_{\mathbf{m}}$ acts $A$ as $K$-algebra automorphisms by

$$
\sigma_{s, t}\left(\left[\begin{array}{c}
X_{1} \\
X_{2} \\
X_{3} \\
X_{4}
\end{array}\right]\right)=\left[\begin{array}{lllll}
s^{-1} & & & \\
& s & & \\
& & s t & \\
& & & t
\end{array}\right]\left[\begin{array}{c}
X_{1} \\
X_{2} \\
X_{3} \\
X_{4}
\end{array}\right] .
$$

Then $\mathcal{Q}\left(A^{G}\right)=K\left(X_{1} X_{2}\right) \subsetneq \mathcal{Q}(A)^{G}=K\left(X_{1} X_{2}, \frac{X_{1} X_{3}}{X_{4}}\right)$.

Lemma 2.2 Let $G$ be a group acting on an integrally closed domain $A$ as ring automorphisms. Let $N$ be a finite normal subgroup of $G$ and $H$ a normal subgroup of $G$ of a finite index (i.e., $[G: H]<\infty)$ such that $N \subseteq H$. Then $\mathcal{Q}\left(A^{G}\right)=\mathcal{Q}(A)^{G}$ if and only if $\mathcal{Q}\left(A^{H}\right)=\mathcal{Q}\left(A^{N}\right)^{H / N}$.

Proof. The if part of the assertion follows easily from finiteness of $G / H$. Suppose that $\mathcal{Q}\left(A^{G}\right)=\mathcal{Q}(A)^{G}$. Let $\widetilde{A^{G}}$ be the integral closure of $A^{G}$ in $\mathcal{Q}(A)^{H}$. As $\mathcal{Q}(A)^{H}$ is a Galois extension over $\mathcal{Q}(A)^{G}$ under the action of $G / H$, we easily see $\mathcal{Q}\left(\widetilde{A^{G}}\right)=\mathcal{Q}(A)^{H}$. Since any element of $\widetilde{A^{G}}$ is integral over $A$ and $A$ is integrally closed, we must have

$$
\widetilde{A^{G}} \subseteq A \cap \mathcal{Q}(A)^{H}=A^{H} .
$$

which shows $\mathcal{Q}\left(A^{H}\right)=\mathcal{Q}(A)^{H}$. Then we see $\mathcal{Q}\left(A^{H}\right)=\mathcal{Q}\left(A^{N}\right)^{H / N}$. 
2.C. An affine scheme $Z=\operatorname{Spec}(R)$ is said to be Krull if $R$ is a Krull domain. Let $\mathrm{v}_{R, \mathfrak{P}}$ denote the discrete valuation of $Z$ defined by the prime ideal $\mathfrak{P} \in \mathrm{Ht}_{1}(R)$. It is well known that, for any subfield $F$ of $\mathcal{Q}(R)$, the subring $R \cap F$ is also Krull. If $R \cap F$ is not a field, the set $\operatorname{Over}_{\mathfrak{p}}(R)$ is known to be non-empty for any $\mathfrak{p} \in \operatorname{Ht}_{1}(R \cap F)$ by the independence theorem of valuations (cf. [7, 3, 2, 5]). The ramification index of $\mathfrak{P}$ over $\mathfrak{p}$ is defined to be

$$
\mathrm{e}(\mathfrak{P}, \mathfrak{p}):=\mathrm{v}_{R, \mathfrak{P}}\left(\mathfrak{p} R_{\mathfrak{P}}\right)
$$

where $\mathfrak{P} \in \operatorname{Over}_{\mathfrak{p}}(R)$. The $p$-part (resp. the $p^{\prime}$-part) (cf. Sect. 1) of e( $\left.\mathfrak{P}, \mathfrak{p}\right)$ stands for $\mathrm{e}_{p}(\mathfrak{P}, \mathfrak{p})\left(\right.$ resp. $\left.\mathrm{e}_{p^{\prime}}(\mathfrak{P}, \mathfrak{p})\right)$.

Lemma 2.3 Let $R$ be a Krull domain acted by a group $G$ as ring automorphisms. Then $R^{G}$ is a Krull domain. In the case where $R^{G}$ is not a field, the set $\operatorname{Over}_{\mathfrak{p}}(R)$ is non-empty for any $\mathfrak{p} \in \mathrm{Ht}_{1}\left(R^{G}\right)$ and the following conditions are equivalent:

(i) $\mathcal{Q}\left(R^{G}\right)=\mathcal{Q}(R)^{G}$.

(ii) $R_{\mathfrak{P}} \cap \mathcal{Q}(R)^{G}=R_{\mathfrak{P} \cap R^{G}}^{G}$ for $\mathfrak{P} \in \operatorname{Ht}_{1}\left(R, R^{G}\right)$.

Proof. As $R^{G}=R \cap \mathcal{Q}(R)^{G}$, the former two assertions follow from the preceding paragraph (cf. [5). Since $R_{\mathfrak{P}}$ with a prime ideal $\mathfrak{P}$ of $R$ of height 1 is a valuation ring of $\mathcal{Q}(R)$, for any nonzero element $x$ of $\mathcal{Q}(R)^{G}, x$ or $x^{-1}$ belongs to $R_{\mathfrak{P}} \cap \mathcal{Q}(R)^{G}$, which shows the implication (ii) $\Rightarrow(i)$.

(i) $\Rightarrow$ (ii) : Suppose that $\mathcal{Q}\left(R^{G}\right)=\mathcal{Q}(R)^{G}$. For any $\mathfrak{P} \in \mathrm{Ht}_{1}\left(R, R^{G}\right), R_{\mathfrak{P}} \cap \mathcal{Q}(R)^{G}$ is a valuation ring of $\mathcal{Q}\left(R^{G}\right)$ contains the discrete valuation $\operatorname{ring} R_{\mathfrak{P} \cap R^{G}}^{G}$ and by Chap. 4 of [6] (e.g. by Chap. 7 of [2]) of the $\operatorname{ring} R_{\mathfrak{P}} \cap \mathcal{Q}(R)^{G}$ is a localization of $R_{\mathfrak{P} \cap R^{G}}^{G}$ at its prime ideal. Unless (ii) holds, we have $R_{\mathfrak{P}} \cap \mathcal{Q}(R)^{G}=\mathcal{Q}\left(R^{G}\right)$. Then as

$$
\mathfrak{P} R_{\mathfrak{P}} \supseteq \mathfrak{P} R_{\mathfrak{P}} \cap \mathcal{Q}(R)^{G} \supseteq \mathfrak{P} \cap R^{G} \neq\{0\},
$$

$\mathfrak{P} R_{\mathfrak{P}}$ contains a unit, which is a contradiction.

Proposition 2.4 Let $A$ be an integrally closed domain acted faithfully by a finite group $H$ as automorphisms and $L$ a subgroup of $H$. Let $\mathfrak{Q}$ be a prime ideal of $A$ and suppose that $A_{\mathfrak{Q} \cap A^{H}}^{H}$ is noetherian. Then the canonical monomorphism

$$
A_{\mathfrak{Q} \cap A^{H}}^{H} \rightarrow A_{\mathfrak{Q} \cap A^{L}}^{L}
$$

is étale if and only if $L \supset \mathcal{I}_{H}(\mathfrak{Q})$.

Proof. Since $A_{\mathfrak{Q} \cap A^{H}}^{H} \otimes_{A^{H}} A$ is an integral closure of the integrally closed domain $A_{\mathfrak{Q} \cap A^{H}}^{H}$ in the finite separable extension $\mathcal{Q}(A)$ over $\mathcal{Q}(A)^{H}$, we see that $A_{\mathfrak{Q} \cap A^{H}}^{H} \otimes_{A^{H}} A$ is finitely generated as an $A_{\mathfrak{Q} \cap A^{H}}^{H}$-module (e.g., Chap. $\mathrm{V}$ of [17]). Exchanging the localization $A_{\mathfrak{Q} \cap A^{H}}^{H} \otimes_{A^{H}} A$ with $A$, we may suppose that the rings $A, A^{L}$ and $A^{H}$ are noetherian and $A$ is finitely generated as an $A^{H}$-module. Then the assertion follows from Proposition 2.2 and Corollary 2.4 of Exposé V of [4.

Corollary 2.5 Let $A$ be a Krull domain acted faithfully by a finite group $H$ as automorphisms and $L$ a subgroup of $H$. For a prime ideal $\mathfrak{Q} \in \mathrm{Ht}_{1}(A)$, the canonical monomorphism

$$
A_{\mathfrak{Q} \cap A^{H}}^{H} \rightarrow A_{\mathfrak{Q} \cap A^{L}}^{L}
$$

is étale if and only if $L \supset \mathcal{I}_{H}(\mathfrak{Q})$.

Proof. Since $A_{\mathfrak{Q} \cap A^{H}}^{H}$ is a discrete valuation ring, this assertion follows from Proposition 2.4 . 
2.D. The pseudo-reflections of finite linear groups and its slight modification are defined and studied in 1, 16, 8]. We generalize the group generated by pseudo-reflections as follows: For a Krull domain $R$ acted by a group $G$ as ring automorphisms, let

$$
\mathfrak{R}(R, G)=\left\langle\bigcup_{\mathfrak{P} \in \mathrm{Ht}_{1}\left(R, R^{G}\right)} \mathcal{I}_{G}(\mathfrak{P})\right\rangle \subseteq G
$$

which is called the pseudo-reflection group of the action of $G$ on $R$.

In the case where $\left.G\right|_{R}$ is finite, for any $\mathfrak{q} \in \mathrm{H} t_{1}\left(R^{G}\right)$, consider $R_{\mathfrak{q}}^{G} \otimes_{R^{G}} R$ with the action of $G$. Then we can apply the ramification theory of Dedekind domains (e.g., Chap. V of [17]) to this action and must have $\mathrm{e}\left(\mathfrak{Q}, \mathfrak{Q} \cap R^{G}\right)=\mathrm{e}\left(\mathfrak{Q}, \mathfrak{Q} \cap R^{\mathcal{I}_{G}(\mathfrak{Q})}\right)$ and

$$
\mathrm{e}_{p^{\prime}}\left(\mathfrak{Q}, \mathfrak{Q} \cap R^{\mathcal{I}_{G}(\mathfrak{Q})}\right)=\sharp_{p^{\prime}}\left(\left.\mathcal{I}_{G}(\mathfrak{Q})\right|_{R}\right)
$$

for $\mathfrak{Q} \in \operatorname{Over}_{\mathfrak{q}}(R)$, where $p$ denotes the characteristic of $R$. The last equality holds in the case where $\left.\mathcal{I}_{G}(\mathfrak{Q})\right|_{R}$ is finite, even if $G$ is not finite on $R$, because $\mathcal{I}_{H}(\mathfrak{Q})=H$ for $H=\mathcal{I}_{G}(\mathfrak{Q})$.

\section{Character Groups and Inertial Quotients}

Hereafter in this paper we suppose that $G$ is an affine algebraic group over $K$. A regular action $(X, G)$ on an affine variety $X$ is said to be stable, if $X$ contains a non-empty open subset consisting of closed $G^{0}$-orbits (or, equivalently, closed $G$-orbits) (cf. [13])

Remark 3.1 Suppose that $G^{0}$ is an algebraic torus and $(X, G)$ is a regular action of $G$ on an affine integral $K$-scheme $X=\operatorname{Spec}(R)$. If

$$
\mathfrak{X}\left(G^{0}\right)^{R}=\mathfrak{X}\left(G^{0}\right)_{R}
$$

(for the notation, see 1.F. of Sect.1), then $\mathcal{Q}\left(R^{G}\right)=\mathcal{Q}(R)^{G}$. Especially in the case where $R$ is finitely generated over $K$ as a $K$-algebra, $(X, G)\left(\right.$ or $\left.\left(X, G^{0}\right)\right)$ is stable if and only if (3.1) holds. If $p=0$, there exists a maximal $G$-invariant $K$-subalgebra $R^{\prime}$ of $R$ such that $R^{G}=R^{\prime G}$ and $\left(X^{\prime}, G\right)$ is stable for $X^{\prime}=\operatorname{Spec}\left(R^{\prime}\right)$ (cf. [11]).

In general if $(X, G)$ is stable, $\mathcal{Q}\left(R^{G}\right)=\mathcal{Q}(R)^{G}$ holds (cf. [13]), but the converse of this assertion does not hold as follows even if $G$ is an algebraic torus.

Example 3.2 The $K$-subalgebra $B=K\left[X_{1}, X_{2}, X_{3}\right]$ of $A$ is invariant under the action of $G=\left(\mathbf{G}_{\mathbf{m}}\right)^{2}$ in Example 2.1 and $\mathcal{Q}\left(B^{G}\right)=\mathcal{Q}(B)^{G}$ holds. However the action $(Y, G)$ is not stable for $Y=\operatorname{Spec}(B)$.

Proposition 3.3 Let $(S, G)$ be a regular action of $G$ on an affine integral $K$-scheme $S=\operatorname{Spec}(A)$. Let $L$ be a finite subgroup of the centralizer $Z_{G}\left(G^{0}\right)$ of $G^{0}$. Then:

(i) $\sharp_{p^{\prime}}\left(\left.L\right|_{A}\right) \cdot \mathfrak{X}\left(G^{0}\right)^{A} \subseteq \mathfrak{X}\left(G^{0}\right)^{A^{L}}$.

(ii) $\sharp_{p^{\prime}}\left(\left.L\right|_{A}\right) \cdot \mathfrak{X}\left(G^{0}\right)_{A} \subseteq \mathfrak{X}\left(G^{0}\right)_{A^{L}}$.

(iii) Both indices $\left[\left\langle\mathfrak{X}\left(G^{0}\right)^{A}\right\rangle:\left\langle\mathfrak{X}\left(G^{0}\right)^{A^{L}}\right\rangle\right]$ and $\left[\mathfrak{X}\left(G^{0}\right)_{A}: \mathfrak{X}\left(G^{0}\right)_{A^{L}}\right]$ are finite and not divisible by $p$ if $p>0$.

Proof. (i): We suppose that $L$ acts faithfully on $A$. For any $\chi \in \mathfrak{X}\left(G^{0}\right)^{A}$, the non-zero $K$-subspace $A_{\chi}$ is invariant under the action of $L$, because $L \subseteq Z_{G}\left(G^{0}\right)$. Let $L_{p}$ denote a $p$-Sylow subgroup of $L$ if $p>0$ and put $L_{p}=\{1\}$ otherwise. Since the group $L_{p}$ is unipotent or trivial, we have a nonzero element $x$ in $A_{\chi} \cap A^{L_{p}}$. Expressing

$$
L=\bigsqcup_{i=1}^{u} \sigma_{i} L_{p} \text { (a disjoint union) }
$$


for $\sigma_{i} \in L\left(1 \leqq i \leqq u=\sharp_{p^{\prime}}(L)\right)$, put

$$
\tilde{x}=\prod_{i=1}^{u} \sigma_{i}(x) \in A_{u \chi} .
$$

Then we easily see that $A^{L} \ni \tilde{x} \neq 0$, which implies $u \chi \in \mathfrak{X}\left(G^{0}\right)^{A^{L}}$ and $(i)$.

The assertion (ii) follows immediately from (i). Since the factor groups

$$
\left\langle\mathfrak{X}\left(G^{0}\right)^{A}\right\rangle /\left\langle\mathfrak{X}\left(G^{0}\right)^{A^{L}}\right\rangle \text { and } \mathfrak{X}\left(G^{0}\right)_{A} / \mathfrak{X}\left(G^{0}\right)_{A^{L}}
$$

are finitely generated, by (i) and (ii) we see that the groups are finite abelian groups with the exponents which are divisors of $\sharp_{p^{\prime}}(L)$. Thus the assertion (iii) follows from this.

Lemma 3.4 Let $\left(S_{i}, G\right)(i=1,2)$ be regular actions of $G$ with an algebraic torus $G^{0}$ on affine integral $K$-schemes $S_{i}=\operatorname{Spec}\left(A_{i}\right)$. If $\mathcal{Q}\left(A_{1}\right)=\mathcal{Q}\left(A_{2}\right)$, then

$$
\left\langle\mathfrak{X}\left(G^{0}\right)^{A_{1}}\right\rangle=\left\langle\mathfrak{X}\left(G^{0}\right)^{A_{2}}\right\rangle .
$$

Proof. Since the assertion is symmetry, we need only to show the inclusion

$$
\mathfrak{X}\left(G^{0}\right)^{A_{2}} \subseteq\left\langle\mathfrak{X}\left(G^{0}\right)^{A_{1}}\right\rangle .
$$

Suppose that $\left(A_{2}\right)_{\psi} \neq\{0\}$ for a character $\psi \in \mathfrak{X}\left(G^{0}\right)$ and let $b$ be a nonzero element of $\left(A_{2}\right)_{\psi}$. Let $\left(A_{1}: b\right)_{A_{1}}$ denote the ideal quotient $\left\{a \in A_{1} \mid a \cdot b \in A_{1}\right\}$. As $b \in \mathcal{Q}\left(A_{1}\right)=$ $\mathcal{Q}\left(A_{2}\right)$, we see that $\left(A_{1}: b\right)_{A_{1}}$ is a nonzero $G^{0}$-invariant ideal of $A_{1}$. This implies that

$$
\left(A_{1}\right)_{\chi} \supseteq\left(\left(A_{1}: b\right)_{A_{1}}\right)_{\chi} \neq\{0\}
$$

for some $\chi \in \mathfrak{X}\left(G^{0}\right)$ and

$$
\left(A_{1}\right)_{\psi+\chi} \supseteq b \cdot\left(\left(A_{1}: b\right)_{A_{1}}\right)_{\chi} \neq\{0\} .
$$

Thus

$$
\psi=(\psi+\chi)-\chi \in\left\langle\mathfrak{X}\left(G^{0}\right)^{A_{1}}\right\rangle,
$$

which shows $\mathfrak{X}\left(G^{0}\right)^{A_{2}} \subseteq\left\langle\mathfrak{X}\left(G^{0}\right)^{A_{1}}\right\rangle$.

Applying Lemma 3.4 to Proposition 3.3, we must have

Proposition 3.5 Let $\left(S_{i}, G\right)(i=1,2)$ be regular actions of $G$ with an algebraic torus $G^{0}$ on affine integral $K$-schemes $S_{i}=\operatorname{Spec}\left(A_{i}\right)$. Let $L$ be a finite subgroup of $Z_{G}\left(G^{0}\right)$. Suppose that $\mathcal{Q}\left(A_{1}^{L}\right)=\mathcal{Q}\left(A_{2}\right)$. Then:

(i) $\sharp_{p^{\prime}}\left(\left.L\right|_{A_{1}}\right) \cdot\left\langle\mathfrak{X}\left(G^{0}\right)^{A_{1}}\right\rangle \subseteq\left\langle\mathfrak{X}\left(G^{0}\right)^{A_{2}}\right\rangle$.

(ii) $\left\langle\mathfrak{X}\left(G^{0}\right)^{A_{1}}\right\rangle$ contains $\left\langle\mathfrak{X}\left(G^{0}\right)^{A_{2}}\right\rangle$ as a subgroup whose index

$$
\left[\left\langle\mathfrak{X}\left(G^{0}\right)^{A_{1}}\right\rangle:\left\langle\mathfrak{X}\left(G^{0}\right)^{A_{2}}\right\rangle\right]
$$

is not divisible by $p$ if $p>0$.

Proposition 3.6 Let $(X, G)$ be a regular action of $G$ with an algebraic torus $G^{0}$ on an affine integral $K$-scheme $X=\operatorname{Spec}(R)$ such that $R$ is integrally closed. Let $L$ be a finite subgroup of $Z_{G}\left(G^{0}\right)$. Let $\mathfrak{P}$ be a $G^{0}$-invariant non-zero prime ideal of $R$. Then $R^{L}$, $R^{\mathcal{I}_{L}(\mathfrak{P})}$ and their reductions modulo by the ideals induced by $\mathfrak{P}$ are naturally $G^{0}$-modules and the following properties hold: 


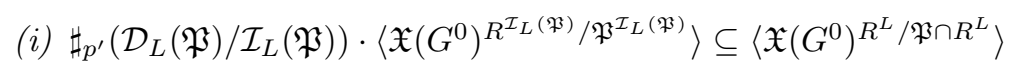

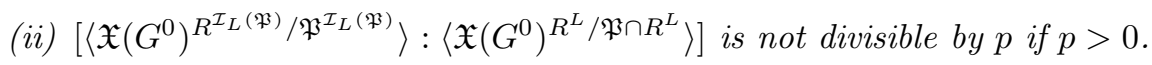

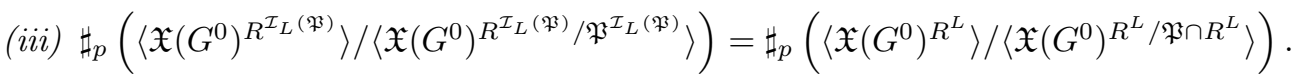

Proof. The first assertion is obvious. Clearly $\left.\mathcal{D}_{L}(\mathfrak{P})\right|_{R}$ is the decomposition group and $\left.\mathcal{I}_{L}(\mathfrak{P})\right|_{R}$ is the inertia group at $\mathfrak{P}$ under the faithful action of $\left.L\right|_{R}$ on $R$. The inclusions

$$
R^{L} \subset R^{\mathcal{D}_{L}(\mathfrak{P})} \subset R^{\mathcal{I}_{L}(\mathfrak{P})}
$$

induce the monomorphisms

$$
\left(R^{L} / \mathfrak{P} \cap R^{L}\right) \hookrightarrow\left(R^{\mathcal{D}_{L}(\mathfrak{P})} / \mathfrak{P} \cap R^{\mathcal{D}_{L}(\mathfrak{P})}\right) \hookrightarrow\left(\left(R^{\mathcal{I}_{L}(\mathfrak{P})} / \mathfrak{P} \cap R^{\mathcal{I}_{L}(\mathfrak{P})}\right)^{\mathcal{D}_{L}(\mathfrak{P}) / \mathcal{I}_{L}(\mathfrak{P})}\right)
$$

By the Galois theory $\left(R, L_{\mid R}\right)$ of an integrally closed domain(cf. Chap. V of [7]), we see

$$
\begin{aligned}
& R_{\mathfrak{P} \cap R^{\mathcal{D}_{L}(\mathfrak{P})}}^{\mathcal{D}_{L}(\mathfrak{P})} /\left(\mathfrak{P} \cap R^{\mathcal{D}_{L}(\mathfrak{P})}\right) R_{\mathfrak{P}^{\prime} \cap R^{\mathcal{D}_{L}(\mathfrak{P})}}^{\mathcal{D}_{L}(\mathfrak{P})}
\end{aligned}
$$

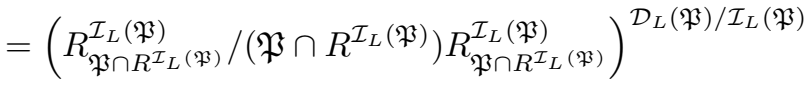

and

$$
R_{\mathfrak{P}^{\prime} \cap R^{L}}^{L} /\left(\mathfrak{P} \cap R^{L}\right) R_{\mathfrak{P}_{\cap} \cap R^{L}}^{L}=R_{\mathfrak{P}_{L} \cap R^{\mathcal{D}_{L}(\mathfrak{P})}}^{\mathcal{D}_{(\mathfrak{P}}} /\left(\mathfrak{P} \cap R^{\mathcal{D}_{L}(\mathfrak{P})}\right) R_{\mathfrak{P}_{L} \cap R^{\mathcal{D}_{L}(\mathfrak{P})}}^{\mathcal{D}_{(\mathfrak{B}}} .
$$

These equalities imply

$$
\begin{aligned}
\mathcal{Q}\left(R^{L} / \mathfrak{P} \cap R^{L}\right) & =\mathcal{Q}\left(R^{\mathcal{D}_{L}(\mathfrak{P})} / \mathfrak{P} \cap R^{\mathcal{D}_{L}(\mathfrak{P})}\right) \\
& =\mathcal{Q}\left(R^{\mathcal{I}_{L}(\mathfrak{P})} / \mathfrak{P} \cap R^{\mathcal{I}_{L}(\mathfrak{P})}\right)^{\mathcal{D}_{L}(\mathfrak{P})} \\
& =\mathcal{Q}\left(\left(R^{\mathcal{I}_{L}(\mathfrak{P})} / \mathfrak{P} \cap R^{\mathcal{I}_{L}(\mathfrak{P})}\right)^{\mathcal{D}_{L}(\mathfrak{P})}\right) .
\end{aligned}
$$

Applying Proposition 3.5 to the replacements

$$
A_{1}=R^{\mathcal{I}_{L}(\mathfrak{P})} / \mathfrak{P} \cap R^{\mathcal{I}_{L}(\mathfrak{P})}, A_{2}=R^{L} / \mathfrak{P} \cap R^{L} \text { and } L=\mathcal{D}_{L}(\mathfrak{P}),
$$

we obtain (i) and see that

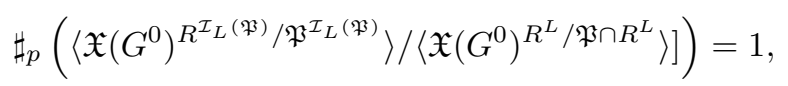

which shows (ii). On the other hand, applying Proposition 3.5 to the replacement

$$
A_{1}=R, A_{2}=R^{L} \text { and } L=L,
$$

we see also that $\left[\left\langle\mathfrak{X}\left(G^{0}\right)^{R}\right\rangle:\left\langle\mathfrak{X}\left(G^{0}\right)^{R^{L}}\right\rangle\right]$ and so

$$
\left[\left\langle\mathfrak{X}\left(G^{0}\right)^{R^{\mathcal{I}_{L}(\mathfrak{P})}}\right\rangle:\left\langle\mathfrak{X}\left(G^{0}\right)^{R^{L}}\right\rangle\right]
$$

are not divisible by $p$ if $p>0$.

By the commutative diagram

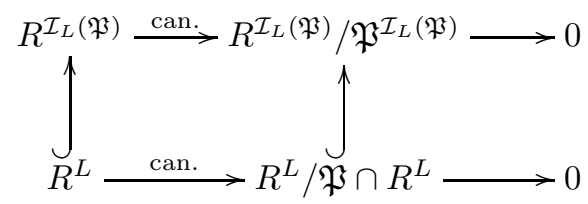


with $G^{0}$-invariant exact rows, we have the descending chains

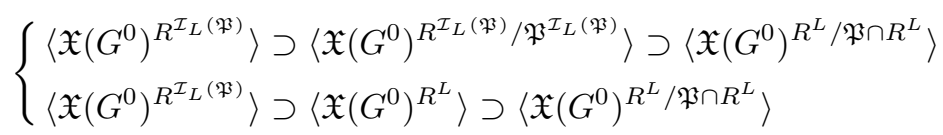

of subgroups. Consequently the assertion (iii) follows from (ii) and the last conclusion of the former paragraph of the proof.

In Sect.6 we use Proposition 3.6 for an affine Krull $K$-scheme $X=\operatorname{Spec}(R)$ and any prime ideal $\mathfrak{P} \in \mathrm{Ht}_{1}\left(R, R^{G}\right)$. In this case, $\mathfrak{P}$ is known to be $G^{0}$-invariant (cf. [5]).

\section{Finite Group Actions on Toric Quotients}

Throughout this section, we suppose $G^{0}$ is an algebraic torus. For a subset $\Delta$ of $G$, let $\operatorname{Unip}(\Delta)$ denote the set consisting of all elements in $\Delta$ which are unipotent in $G$.

Proposition 4.1 Let $\Gamma$ be a subgroup $\left\langle\operatorname{Unip}(\Gamma) \cup \Gamma_{0}\right\rangle$ of $G$ for a finite subset $\Gamma_{0}$ of $G$ of finite orders. If $G=Z_{G}\left(G^{0}\right)$, then $\Gamma$ is finite.

Proof. Let $\rho: G \rightarrow G L(V)$ be a faithful finite dimensional rational representation of $G$. The $K$-space $V$ is a direct sum $\bigoplus_{i=1}^{n} V_{\chi_{i}}$ of non-zero subspaces $V_{\chi_{i}}$ of $V$ of relative invariants (cf. 1.F.) with respect to distinct characters $\chi_{i} \in \mathfrak{X}\left(G^{0}\right)$. As $G \subseteq Z_{G}\left(G^{0}\right)$, each subspace $V_{\chi_{i}}(1 \leq i \leq n)$ is invariant under the action of $G$ on $V$. Let

$$
\rho_{i}: G \rightarrow G L\left(V_{\chi_{i}}\right)
$$

denote the representation defined in a natural way. Since each element of $\rho_{i}(\operatorname{Unip}(G))$ is unipotent on $V_{\chi}$, we have

$$
\operatorname{det}_{V_{\chi_{i}}}\left(\rho_{i}(\langle\operatorname{Unip}(G)\rangle)\right)=1
$$

where $\operatorname{det}_{V_{\chi_{i}}}$ denotes the determinant on $V_{\chi}$. From this observation and the assumption on generators of $\Gamma$, we infer that the image of the morphism

$$
\operatorname{det}_{V_{\chi_{i}}}: \rho_{i}(\Gamma) \longrightarrow K^{\times}
$$

is finite. For a nontrivial character $\chi_{i}$, as $\rho_{i}\left(G^{0}\right)$ is of rank one and the morphism

$$
\operatorname{det}_{V_{\chi_{i}}}: \rho_{i}\left(G^{0}\right) \rightarrow K^{\times}
$$

is surjective, we see that

$$
\operatorname{Ker}\left(\rho_{i}\left(G^{0}\right) \underset{\operatorname{det}_{\chi_{\chi_{i}}}}{\longrightarrow} K^{\times}\right)
$$

is a finite group. Consequently the kernel and image of morphism

$$
\rho_{i}\left(G^{0} \cap \Gamma\right) \underset{\operatorname{det}_{V_{\chi_{i}}}}{\longrightarrow} K^{\times}
$$

are finite, which imply $\rho_{i}\left(G^{0} \cap \Gamma\right)$ is a finite for a nontrivial $\chi_{i}$. As $\rho_{i}(\Gamma) / \rho_{i}\left(G^{0} \cap \Gamma\right)$ is the epimorphic image of the finite group $\Gamma / G^{0} \cap \Gamma$, it is finite if $\chi_{i}$ is nontrivial. On the other hand, if $\chi_{i}$ is the trivial character, $\rho_{i}(G)$ is clearly finite. Because $\Gamma$ can be embedded in the product

$$
\prod_{i=1}^{n} \rho_{i}(\Gamma)
$$

of finite groups, we conclude that $\Gamma$ is also finite. 
Notation Hereafter in this section, let $(X, G)$ denote a regular effective action of $G$ on an affine Krull $K$-scheme $X=\operatorname{Spec}(R)$. Clearly $\left.G\right|_{R^{G}}$ is a finite group. Hence, let $\mathcal{I}_{G}(\mathfrak{q})_{[p]}$ denote the inverse image of a $p$-Sylow subgroup of $\left.\mathcal{I}_{G}(\mathfrak{q})\right|_{R^{G^{0}}}$ under the canonical homomorphism

$$
\left.\mathcal{I}_{G}(\mathfrak{q}) \rightarrow \mathcal{I}_{G}(\mathfrak{q})\right|_{R^{G^{0}}}
$$

for any $\mathfrak{q} \in \mathrm{Ht}_{1}\left(R^{G^{0}}\right)$ if $p(=\operatorname{char}(K))>0$. Otherwise put

$$
\mathcal{I}_{G}(\mathfrak{q})_{[0]}=\operatorname{Ker}\left(\left.\mathcal{I}_{G}(\mathfrak{q}) \rightarrow \mathcal{I}_{G}(\mathfrak{q})\right|_{R^{G^{0}}}\right) .
$$

Both groups $\mathcal{I}_{G}(\mathfrak{q})$ and $\mathcal{I}_{G}(\mathfrak{q})_{[p]}$ contain $G^{0}$ and are closed in $G$.

Lemma 4.2 Let $\mathfrak{q}$ be any prime ideal in $\operatorname{Ht}_{1}\left(R^{G^{0}}\right)$. Then $\mathcal{I}_{G}(\mathfrak{q})_{[p]}$ is normal in $\mathcal{I}_{G}(\mathfrak{q})$ and $\mathcal{I}_{G}(\mathfrak{q}) / \mathcal{I}_{G}(\mathfrak{q})_{[p]}$ is a subgroup of of $\mathrm{U}\left(R_{\mathfrak{q}}^{G^{0}} / \mathfrak{q} R_{\mathfrak{q}}^{G^{0}}\right)$.

Proof. Recalling $\mathcal{I}_{G}(\mathfrak{q})=\left\{\sigma \in G \mid(\sigma-1)\left(R^{G^{0}}\right) \subseteq \mathfrak{q}\right\}$, we see

$$
\left.\mathcal{I}_{G}(\mathfrak{q})\right|_{R^{G^{0}}}=\mathcal{I}_{\left.G\right|_{R} G^{0}}(\mathfrak{q})=\mathcal{I}_{\left.G\right|_{R^{0}}}\left(R_{R^{G} \cap \mathfrak{q}}^{G} \otimes_{R^{G}} \mathfrak{q}\right)
$$

which is the inertia group of $R_{R^{G} \cap \mathfrak{q}}^{G} \otimes_{R^{G}} \mathfrak{q}$ for the finite Galois group action

$$
\left(R_{R^{G} \cap \mathfrak{q}}^{G} \otimes_{R^{G}} R^{G^{0}},\left.G\right|_{R^{G^{0}}}\right)
$$

on the semilocal Dedekind domain. We now examine and apply the proof of Theorem 25 in $\S 10$ of Chap. V of [17] to our case. Then the $p$-Sylow subgroup $H_{1}$ of $\left.\mathcal{I}_{G}(\mathfrak{q})\right|_{R^{G^{0}}}=$ $\mathcal{I}_{\left.G\right|_{R^{G}}}\left(R_{R^{G} \cap \mathfrak{q}}^{G} \otimes_{R^{G}} \mathfrak{q}\right)$ is normal if $p>0$. Regarding $H_{1}$ is trivial in the case where $p=0$ we always have

$$
\left(\left.\mathcal{I}_{G}(\mathfrak{q})\right|_{R^{G^{0}}}\right) / H_{1} \hookrightarrow \mathrm{U}\left(R_{\mathfrak{q}}^{G^{0}} / \mathfrak{q} R_{\mathfrak{q}}^{G^{0}}\right)
$$

as groups for any characteristic $p$. Thus the assertion follows from this observation.

Proposition 4.3 Suppose that $G=Z_{G}\left(G^{0}\right)$. For any $\mathfrak{q} \in \operatorname{Ht}_{1}\left(R^{G^{0}}\right)$, we have:

(i) $\left\langle\operatorname{Unip}\left(\mathcal{I}_{G}(\mathfrak{q})\right)\right\rangle$ is a finite group satisfying $\left.\left\langle\operatorname{Unip}\left(\mathcal{I}_{G}(\mathfrak{q})\right)\right\rangle\right|_{R^{G^{0}}}=\left.\mathcal{I}_{G}(\mathfrak{q})_{[p]}\right|_{R^{G^{0}}}$ and

$$
R^{\left\langle\operatorname{Unip}\left(\mathcal{I}_{G}(\mathfrak{q})\right)\right\rangle \cdot G^{0}}=R^{\mathcal{I}_{G}(\mathfrak{q})_{[p]}} .
$$

(ii) There exists a diagonalizable closed subgroup, denoted by $\mathcal{D}_{G, \mathfrak{q}}$, of $\mathcal{I}_{G}(\mathfrak{q})$ such that $D_{G, \mathfrak{q}} \supseteq G^{0}, \mathcal{D}_{G, \mathfrak{q}} / G^{0}$ is cyclic, and

$$
R^{\left\langle\operatorname{Unip}\left(\mathcal{I}_{G}(\mathfrak{q})\right)\right\rangle \cdot \mathcal{D}_{G, \mathfrak{q}}}=R^{\mathcal{I}_{G}(\mathfrak{q})} .
$$

(iii) $R_{\mathfrak{q} \cap R^{G}}^{G} \rightarrow R_{\mathfrak{q} \cap R^{I_{G}(\mathfrak{q})}}^{\mathcal{I}_{G}(\mathfrak{q})}$ is étale and the ramification index

$$
\mathrm{e}\left(\mathfrak{q} \cap R^{\left\langle\operatorname{Unip}\left(\mathcal{I}_{G}(\mathfrak{q})\right)\right\rangle \cdot G^{0}}, \mathfrak{q} \cap R^{G}\right)
$$

is not divisible by $p$ if $p>0$.

Proof. (i): By Proposition 4.1 we easily see that the group $\langle\operatorname{Unip}(G)\rangle$ is of finite order. Let $\sigma \in \mathcal{I}_{G}(\mathfrak{q})_{[p]}$ and express $\sigma=\sigma_{u} \cdot \sigma_{s}$ for a unipotent (resp. semisimple) element $\sigma_{u}$ (resp. $\left.\sigma_{s}\right)$ in $\mathcal{I}_{G}(\mathfrak{q})_{[p]}$. As $\left.\sigma\right|_{R^{G^{0}}}$ is unipotent, we see

$$
\left.\sigma\right|_{R^{G^{0}}}=\left.\left.\sigma_{u}\right|_{R^{G^{0}}} \in \operatorname{Unip}\left(\mathcal{I}_{G}(\mathfrak{q})\right)\right|_{R^{G^{0}}} .
$$

On the other hand, the inclusion

$$
\left.\left.\operatorname{Unip}\left(\mathcal{I}_{G}(\mathfrak{q})\right)\right|_{R^{G^{0}}} \subseteq \mathcal{I}_{G}(\mathfrak{q})_{[p]}\right|_{R^{G^{0}}}
$$


follows, since the number of $p$-Sylow subgroups of $\left.\mathcal{I}_{G}(\mathfrak{q})\right|_{R^{G^{0}}}$ is one (cf. Lemma 4.2) if $p>0$. Consequently

$$
\left.\left\langle\operatorname{Unip}\left(\mathcal{I}_{G}(\mathfrak{q})\right)\right\rangle\right|_{R^{G^{0}}}=\left.\mathcal{I}_{G}(\mathfrak{q})_{[p]}\right|_{R^{G^{0}}}
$$

holds. From this we immediately derive the second equality of $(i)$.

(ii): According to Lemma 4.2, choose $\tau \in \mathcal{I}_{G}(\mathfrak{q})$ in such a way that $\tau$ generates the factor group

$$
\left.I_{G}(\mathfrak{q})\right|_{R^{G^{0}}} /\left.\left(\mathcal{I}_{G}(\mathfrak{q})[p]\right)\right|_{R^{G^{0}}}
$$

on $R^{G^{0}}$ principally. Expressing $\tau=\tau_{u} \cdot \tau_{s}$ with its unipotent part $\tau_{u}$ and semisimple one $\tau_{s}$, we may assume $\tau$ is semisimple. Define $\mathcal{D}_{G, \mathfrak{q}}$ to be the subgroup $\left\langle G^{0}, \tau\right\rangle$. We must have

$$
R^{\left\langle\operatorname{Unip}\left(\mathcal{I}_{G}(\mathfrak{q})\right)\right\rangle \cdot \mathcal{D}_{G, \mathfrak{q}}}=\left(R^{G^{0}}\right)^{\left\langle\operatorname{Unip}\left(\mathcal{I}_{G}(\mathfrak{q})\right)\right\rangle \cdot \mathcal{D}_{G, \mathfrak{q}} / G^{0}}=\left(R^{G^{0}}\right)^{\mathcal{I}_{G}(\mathfrak{q}) / G^{0}}=R^{\mathcal{I}_{G}(\mathfrak{q})}
$$

since $\left.\left\langle\operatorname{Unip}\left(\mathcal{I}_{G}(\mathfrak{q})\right)\right\rangle\right|_{R^{G^{0}}}=\left.\mathcal{I}_{G}(\mathfrak{q})_{[p]}\right|_{R^{G^{0}}}$.

(iii): By the Galois theory on $R^{G^{0}}$ with the finite group $G / G^{0}$ action and Corollary 2.5, we see that

$$
R^{G} \rightarrow R^{\mathcal{I}_{G}(\mathfrak{q})}
$$

is étale at $\mathfrak{q} \cap R^{\mathcal{I}_{G}(\mathfrak{q})}$. Suppose that $p>0$ and consider

$$
\left(R^{\left\langle\operatorname{Unip}\left(\mathcal{I}_{G}(\mathfrak{q})\right)\right\rangle \cdot G^{0}}\right)_{\mathfrak{q} \cap R^{\left\langle\operatorname{Unip}\left(\mathcal{I}_{G}(\mathfrak{q})\right)\right\rangle \cdot G^{0}}}
$$

acted by $\mathcal{D}_{G, \mathfrak{q}} / G^{0}$ in (ii) of this proposition. Applying the ramification theory of Dedekind domains (e.g., Chap. V of [17]) to this discrete valuation ring,

$$
\mathrm{e}\left(\mathfrak{q} \cap R^{\left\langle\operatorname{Unip}\left(\mathcal{I}_{G}(\mathfrak{q})\right)\right\rangle \cdot G^{0}}, \mathfrak{q} \cap R^{\mathcal{I}_{G}(\mathfrak{q})}\right)
$$

is a divisor of the order of the group

$$
\left.\left(\mathcal{D}_{G, \mathfrak{q}} / G^{0}\right)\right|_{R^{\left\langle\operatorname{Unip}\left(\mathcal{I}_{G}(\mathfrak{q})\right)\right\rangle \cdot G^{0}},},
$$

which is not divisible by $p$. Thus the assertion (iii) follows from this.

\section{Towards Krull Schemes}

We now generalize the auxiliary results in Sec. 3 of $[10$ for affine normal varieties to affine Krull $K$-schemes. Stability of actions $(X, G)$ with tori $G^{0}$ can be replaced by $\mathcal{Q}\left(\mathcal{O}(X)^{G}\right)=\mathcal{Q}(\mathcal{O}(X))^{G}$.

Moreover we need in the last section the following theorem which is the main result of [12] and implies that any inertia group $\mathcal{I}_{G}(\mathfrak{Q})$ at $\mathfrak{Q} \in \mathrm{Ht}_{1}\left(\mathcal{O}(X), \mathcal{O}(X)^{G}\right)$ is finite on $X$ for a regular action $(X, G)$ of a reductive algebraic group $G$ on an arbitrary affine Krull $K$-scheme $X$. Pseudo-reflection groups $\mathfrak{R}(\mathcal{O}(X), G)$ of actions are referred to the paragraph 2.D. of Sect. 2.

Theorem 5.1 (cf. [12]) In general, the following conditions are equivalent:

(i) $G$ is (geometrically) reductive.

(ii) For any regular action $(X, G)$ of $G$ on any affine Krull $K$-scheme $X$, the image of the pseudo-reflection group $\mathfrak{R}(\mathcal{O}(X), G)$ to Aut $X$ (i.e., $\left.\left.\mathfrak{R}(\mathcal{O}(X), G)\right|_{\mathcal{O}(X)}\right)$ is finite.

The next result, which is a slight generalization of Proposition 1.8 of [10], can be shown as in the proof in 10 without the assumption that $G^{0}$ is linearly reductive and $X$ is noetherian by the use of Theorem 5.1 and Corollary 2.5. 
Proposition 5.2 (cf. [10]) Suppose that $G$ is a reductive affine algebraic and that $X$ is an affine Krull $K$-scheme with a regular action $(X, G)$ of $G$. Set $Y=X / / \mathfrak{R}(\mathcal{O}(X), G)$. Then:

(i) $\mathfrak{R}(\mathcal{O}(Y), G)=\mathfrak{R}(\mathcal{O}(X), G)$.

(ii) $\left.\mathcal{I}_{G}(\mathfrak{q})\right|_{Y}=\{1\}$ for $\mathfrak{q} \in \mathrm{Ht}_{1}\left(\mathcal{O}(Y), \mathcal{O}(Y)^{G}\right)$.

Proposition 5.3 Let $D$ be a diagonalizable affine algebraic group and $(X, D)$ a regular action of $D$ on an affine Krull K-scheme $X=\operatorname{Spec}(R)$ such that $\mathcal{Q}\left(R^{D}\right)=\mathcal{Q}(R)^{D}$. Let $\mathfrak{Q}$ be a prime ideal in $\operatorname{Ht}_{1}\left(R, R^{D}\right)$. If $\mathfrak{Q}$ is invariant under the action of $D$, then $\left\langle\mathfrak{X}(D)^{R / \mathfrak{Q}}\right\rangle$ is a subgroup of $\left\langle\mathfrak{X}(D)^{R}\right\rangle$ and the quotient group

$$
\left\langle\mathfrak{X}(D)^{R}\right\rangle /\left\langle\mathfrak{X}(D)^{R / \mathfrak{Q}}\right\rangle
$$

is a cyclic group of order equal to the ramification index $\mathrm{e}\left(\mathfrak{Q}, \mathfrak{Q} \cap R^{D}\right)$.

Proof. Since $\mathfrak{Q}$ is a rational $D$-submodule and $D$ is linearly reductive, for each $\chi \in$ $\mathfrak{X}(D)^{R / \mathfrak{Q}}$ we have $R_{\chi} \neq\{0\}$, which implies the first assertion and the equality

$$
\mathfrak{X}(D)^{R}=\mathfrak{X}(D)^{\mathfrak{Q}} \cup \mathfrak{X}(D)^{R / \mathfrak{Q}} .
$$

Let $f$ be an element of $\mathfrak{Q}$ to satisfy $f \cdot R_{\mathfrak{Q}}=\mathfrak{Q} R_{\mathfrak{Q}}$. As there is a finite dimensional rational $D$-submodule $U$ of $\mathfrak{Q}$ containing $f$, exchanging $f$, we can choose the element $f$ in such a way that $f \in R_{\psi}$ for some $\psi \in \mathfrak{X}(D)^{R}$. Let $\mu$ be a character in $\mathfrak{X}(D)^{\mathfrak{Q}}$. Then we have a nonzero element $y \in \mathfrak{Q}$ such that $y \in R_{\mu}$. There are nonzero elements $g, h \in R \backslash \mathfrak{Q}$ satisfying

$$
y=f^{\mathrm{v}_{R, \mathfrak{Q}}(y)} \cdot \frac{g}{h} .
$$

Express the finite sums

$$
g=\sum_{\xi \in \mathfrak{X}(D)} g_{\xi}\left(g_{\xi} \in R_{\xi}\right) \text { and } h=\sum_{\xi \in \mathfrak{X}(D)} h_{\xi}\left(h_{\xi} \in R_{\xi}\right) .
$$

Then $h_{\xi_{1}} \notin \mathfrak{Q}$ for some $\xi_{1} \in \mathfrak{X}(D)$. Clearly by (5.2) we have

$$
y \cdot h_{\xi_{1}}=f^{v_{R, \mathfrak{Q}}(y)} \cdot g_{\xi_{2}}
$$

for some $\xi_{2} \in \mathfrak{X}(D)$. Comparing the values of $\mathrm{v}_{R, \mathfrak{Q}}$ of the above equality, we must have $g_{\xi_{2}} \notin \mathfrak{Q}$, which implies

$$
\mu \equiv v_{R, \mathfrak{Q}}(y) \psi \quad \bmod \left\langle\mathfrak{X}(D)^{R / \mathfrak{Q}}\right\rangle
$$

because $\xi_{1}, \xi_{2} \in \mathfrak{X}(D)^{R / \mathfrak{Q}}$. Consequently by (5.1) we see

$$
\left\langle\mathfrak{X}(D)^{R}\right\rangle /\left\langle\mathfrak{X}(D)^{R / \mathfrak{Q}}\right\rangle
$$

is a cyclic group generated by $\psi+\left\langle\mathfrak{X}(D)^{R / \mathfrak{Q}}\right\rangle$.

By Lemma 2.3, we have $R_{\mathfrak{Q}} \cap \mathcal{Q}(R)^{D}=R_{\mathfrak{Q} \cap R^{D}}^{D}$ and

$$
f R_{\mathfrak{D}} \cap \mathcal{Q}(R)^{D}=\mathfrak{Q} R_{\mathfrak{Q}} \cap \mathcal{Q}(R)^{D}=\left(\mathfrak{Q} \cap R^{D}\right) R_{\mathfrak{Q} \cap R^{D}}^{D} .
$$

From $\mathrm{v}_{R, \mathfrak{Q}}\left(\mathfrak{Q} \cap R^{D}\right)=\mathrm{e}\left(\mathfrak{Q}, \mathfrak{Q} \cap R^{D}\right)$, we infer that

$$
\mathrm{e}\left(\mathfrak{Q}, \mathfrak{Q} \cap R^{D}\right)=\min \left\{k \in \boldsymbol{N} \mid \exists g, h \in R \backslash \mathfrak{Q} \text { such that } f^{k} \cdot \frac{g}{h} \in \mathcal{Q}(R)^{D}\right\}
$$

As in the preceding paragraph, this can be replaced by

$$
\mathrm{e}\left(\mathfrak{Q}, \mathfrak{Q} \cap R^{D}\right)=\min \left\{\begin{array}{l|l}
k \in \boldsymbol{N} & \begin{array}{c}
\exists \text { relative invariants } g, h \in R \backslash \mathfrak{Q} \text { of } D \\
\text { such that } f^{k} \cdot \frac{g}{h} \in \mathcal{Q}(R)^{D}
\end{array}
\end{array}\right\}
$$


(see 1.F. in Sect. 1 for relative invariants of $D$ ). For a rational character $\chi \in\left\langle\mathfrak{X}(D)^{R}\right\rangle$, $\chi \in\left\langle\mathfrak{X}(D)^{R / \mathfrak{Q}}\right\rangle$ if and only if

$$
\begin{aligned}
\chi & =\sum_{\exists\left\{\xi_{i}\right\} \subset \mathfrak{X}(D)^{R / \mathfrak{Q}}} \xi_{i}-\sum_{\exists\left\{\nu_{j}\right\} \subset \mathfrak{X}(D)^{R / \mathfrak{Q}}} \nu_{j} \quad \text { (finite sum) } \\
& =\xi-\nu \quad\left(\exists \xi, \nu \in \mathfrak{X}(D)^{R / \mathfrak{Q}}\right),
\end{aligned}
$$

because the integral domain $R / \mathfrak{Q}$ implies that $\mathfrak{X}(D)^{R / \mathfrak{Q}}$ is a semi-group. Thus the right hand side of (5.3) can be identified with

$$
\min \left\{k \in \boldsymbol{N} \mid k \mu \in\left\langle\mathfrak{X}(D)^{R / \mathfrak{Q}}\right\rangle\right\},
$$

which is equal to the order of the group $\left\langle\mathfrak{X}(D)^{R}\right\rangle /\left\langle\mathfrak{X}(D)^{R / \mathfrak{Q}}\right\rangle$.

If a regular action $(X, G)$ is effective (cf. Sect. 1),

$$
\operatorname{Ker}(G \rightarrow \text { Aut } X) \subseteq Z_{G}\left(G^{0}\right)
$$

follows from its finiteness. A variety $W$ is said to be conical if $X$ is affine and $\mathcal{O}(W)$ is a graded $K$-algebra equipped with a positive gradation $\mathcal{O}(W)=\oplus_{i \geq 0} \mathcal{O}(W)_{i}$ such that $\mathcal{O}(W)_{0}=K$. In this case a regular action $(W, G)$ is said to be conical, if it is regular and the induced action of $G$ on $\mathcal{O}(W)$ preserves the gradation of $\mathcal{O}(W)$. The following result is the restatement of Proposition 2.2 of [10] for $W=\tilde{V} / / L$ in this proposition.

Proposition 5.4 (cf. [10], Propositions 2.2) Suppose that $G^{0}$ is an algebraic torus. Then there exists an effective conical stable action $(W, G)$ of on a conical normal variety $W$ such that $\left(W / / G^{0}, G\right)$ can be identified with a trivial action on the affine line $\boldsymbol{A}^{1}$.

Proposition 5.5 (cf. [10], Proposition 2.3) Suppose that $G^{0}$ is an algebraic torus and $G \neq Z_{G}\left(G^{0}\right)$. If $p=0$ or $\sharp\left(G / Z_{G}\left(G^{0}\right)\right)$ is relatively prime to $p$ for $p>0$, then there exists a closed subgroup $H$ of $G$ containing $Z_{G}\left(G^{0}\right)$ and an effective regular action $(X, H)$ of $H$ on an affine normal variety $X$ such that

$$
\mathrm{e}_{p^{\prime}}\left(\mathfrak{P}, \mathfrak{P} \cap \mathcal{O}(X)^{H}\right) \neq \sharp_{p^{\prime}}\left(\left.\mathcal{I}_{H}(\mathfrak{P})\right|_{X}\right) .
$$

Proof. We choose a closed subgroup $H$ of $G$ in such a way that $H / Z_{G}\left(G^{0}\right)$ is non-trivial cyclic subgroup which is regarded as a subgroup of the multiplicative group $\mathbf{G}_{\mathbf{m}}$ over $K$. Consider the canonical regular action $\left(Y, \mathbf{G}_{\mathbf{m}}\right)$ with $Y=\operatorname{Spec}\left(K\left[X_{1}\right]\right)$ on the affine line $\boldsymbol{A}^{1}$ such as $t\left(X_{1}\right)=t X_{1}\left(t \in \mathbf{G}_{\mathbf{m}}\right)$. By this we define a regular action $\left(Y, H / Z_{G}\left(G^{0}\right)\right)$. Applying Proposition 2.3 to $\left(Y, H / Z_{G}\left(G^{0}\right)\right)$, we obtain an effective regular action $(X, H)$ on an affine normal variety $X$ as desired.

Using Proposition 5.3 and Proposition 5.2 instead of Lemma 3.1 (1) and Proposition 1.8 of [10] respectively, we can similarly show the following result which is a slight generalization of Proposition 3.2 of [10].

Proposition 5.6 Suppose that $G^{0}$ is an algebraic torus and $G=Z_{G}\left(G^{0}\right)$. Let $(X, G)$ be a regular action of $G$ on an affine Krull K-scheme $X=\operatorname{Spec}(R)$ such that $\mathcal{Q}(R)^{G}=$ $\mathcal{Q}\left(R^{G}\right)$. Then $\mathrm{e}\left(\mathfrak{Q}, \mathfrak{Q} \cap R^{G}\right)$ is equal to

$$
\sharp_{p^{\prime}}\left(\left.\mathcal{I}_{G}(\mathfrak{Q})\right|_{R}\right) \cdot \sharp_{p}\left(\left\langle\mathfrak{X}\left(G^{0}\right)^{R}\right\rangle /\left\langle\mathfrak{X}\left(G^{0}\right)^{R / \mathfrak{Q}}\right\rangle\right) \cdot \mathrm{e}_{p}\left(\mathfrak{Q} \cap R^{G^{0}}, \mathfrak{Q} \cap R^{\mathcal{I}_{G}\left(\mathfrak{Q} \cap G^{0}\right)}\right)
$$

for any $\mathfrak{Q} \in \mathrm{Ht}_{1}\left(R, R^{G}\right)$. 


\section{Main Theorem}

As a matter of convenience, we restate Theorem 1.1 as follows:

Theorem 6.1 Suppose that $G^{0}$ is an algebraic torus. Then the following conditions are equivalent:

(i) $G=Z_{G}\left(G^{0}\right)$

(ii) For an arbitrary closed subgroup $H$ of $G$ containing $Z_{G}\left(G^{0}\right)$, the following conditions hold for any effective regular action $(X, H)$ on an arbitrary affine Krull $K$-scheme $X=\operatorname{Spec}(R)$ such that $\mathcal{Q}\left(R^{G^{0}}\right)=\mathcal{Q}(R)^{G^{0}}$ : for any $\mathfrak{P} \in \operatorname{Ht}_{1}\left(R, R^{H}\right)$,

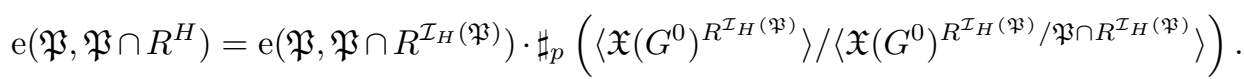

(iii) For an arbitrary closed subgroup $H$ of $G$ containing $Z_{G}\left(G^{0}\right)$, the conditions in (ii) hold for any effective stable regular action $(X, H)$ on an arbitrary affine normal variety $X=\operatorname{Spec}(R)$.

Proof. Since $(X, H)$ in (iii) is a special case mentioned in (ii) (cf. Remark 3.1), the implication (ii) $\Rightarrow$ (iii) is obvious. Note that $Z_{H}\left(G^{0}\right)=Z_{G}\left(G^{0}\right)$ for a subgroup $H$ of $G$ containing $Z_{G}\left(G^{0}\right)$ and that in (ii) the equivalence

$$
\mathcal{Q}\left(\mathcal{O}(X)^{G^{0}}\right)=\mathcal{Q}(\mathcal{O}(X))^{G^{0}} \Longleftrightarrow \mathcal{Q}\left(\mathcal{O}(X)^{H}\right)=\mathcal{Q}(\mathcal{O}(X))^{H}
$$

follows from Lemma 2.2. Moreover in (ii) note that $\mathcal{I}_{H}(\mathfrak{P})$ is a finite group.

(iii) $\Rightarrow($ i $)$ : Assume that this implication is false. Suppose $G$ is a counter-example for (iii) $\Rightarrow\left(\right.$ i), i.e., the condition (iii) holds for $G$ but $G \neq Z_{G}\left(G^{0}\right)$. By (iii) and (2.1) we always have

$$
\mathrm{e}_{p^{\prime}}\left(\mathfrak{P}, \mathfrak{P} \cap \mathcal{O}(X)^{H}\right)=\mathrm{e}_{p^{\prime}}\left(\mathfrak{P}, \mathfrak{P} \cap R^{\mathcal{I}_{H}(\mathfrak{P})}\right)=\sharp_{p^{\prime}}\left(\left.\mathcal{I}_{H}(\mathfrak{P})\right|_{X}\right)
$$

under the circumstances as in (ii). The second equality follows from (2.1), since $\mathcal{I}_{H}(\mathfrak{P})$ is finite on $X$. From Proposition 5.5 we see $p>0$ and the set $G \backslash Z_{G}\left(G^{0}\right)$ contains an element $\tau$ such that order of the coset $\tau Z_{G}\left(G^{0}\right)$ in the group $G / Z_{G}\left(G^{0}\right)$ is equal to $p$.

Let

$$
\phi: C_{1} \rightarrow C_{2}
$$

be a Galois cover of smooth projective curves defined over $K$ which is wildly ramified at a closed point (e.g., [15]). Exchanging $C_{1}$ with its quotient by the normal subgroup of the Galois group of index $p$, we may suppose that the Galois group of $\phi$ is equal to

$$
\Gamma \cong \boldsymbol{Z} / p \boldsymbol{Z}
$$

Then there exists a closed point $P \in C_{1}$ fixing by $\Gamma$, because the inertia group $\mathcal{I}_{\Gamma}(P)$ of $P$ under the action of $\Gamma$ must be equal to $\Gamma$. Let $Y$ be an affine open set containing the closed point $\phi(P)$. Let $H$ be the closed subgroup of $G$ generated by $Z_{G}\left(G^{0}\right)$ and $\tau$ whose quotient group

$$
H / Z_{G}\left(G^{0}\right) \cong \Gamma
$$

acting regularly on the affine normal variety $\phi^{-1}(Y)$. So $\phi^{-1}(Y) / / H=Y$. Let $\mathfrak{P}_{Y}$ be the maximal ideal of $\mathcal{O}\left(\phi^{-1}(Y)\right)$ associated with the point $P \in \phi^{-1}(Y)$. Since $\sharp\left(\left.H\right|_{\phi^{-1}(Y)}\right)=$ $\sharp\left(\left.\mathcal{I}_{H}\left(\mathfrak{P}_{Y}\right)\right|_{\phi^{-1}(Y)}\right)=\sharp(\Gamma)=p$ and $\mathcal{O}\left(\phi^{-1}(Y)\right) / \mathfrak{P}_{Y}=\mathcal{O}(Y) /\left(\mathfrak{P}_{Y} \cap \mathcal{O}(Y)\right)=K$, by ramification theory (e.g., Chap. V of [17]) we see that $\mathrm{e}\left(\mathfrak{P}_{Y}, \mathfrak{P}_{Y} \cap \mathcal{O}(Y)\right)=p$. From Proposition 5.4 there exists a conical effective action of $H$ on a conical normal variety $W$ such that $\left(W / / G^{0}, H\right)$ can be identified with a trivial action on the affine line $\boldsymbol{A}^{1}$. Consider the affine normal variety

$$
X:=\phi^{-1}(Y) \times_{K} W
$$


with $\mathcal{O}(X)=\mathcal{O}\left(\phi^{-1}(Y)\right) \otimes_{K} \mathcal{O}(W)$ on which $H$ acts diagonally. As $H^{0}=G^{0}$ acts on $\phi^{-1}(Y)$ trivially, the regular action $(X, H)$ is stable. Put

$$
\mathfrak{P}:=\mathfrak{P}_{Y} \otimes_{K} \mathcal{O}(W) \in \operatorname{Ht}_{1}(\mathcal{O}(X)) .
$$

Since

$$
\mathcal{O}(X) / \mathfrak{P} \cong \mathcal{O}(W)
$$

is $\mathcal{D}_{H}(\mathfrak{P})$-invariant (cf. 2.B. of Sect. 2), from the definition of inertia groups and (5.4) we must have

$$
\mathcal{I}_{H}(\mathfrak{P}) \subseteq \operatorname{Ker}(H \rightarrow \text { Aut } W) \subseteq Z_{H}\left(G^{0}\right) .
$$

As the action of $Z_{H}\left(G^{0}\right)$ on $\phi^{-1}(Y)$ is trivial, by (6.2) we see

$$
\left.\mathcal{I}_{H}(\mathfrak{P})\right|_{X}=\{1\} .
$$

On the other hand the triviality of $Z_{H}\left(G^{0}\right)$ on $\phi^{-1}(Y)$ and $H$ on $W / / G^{0} \cong \boldsymbol{A}^{1}$ implies the commutative diagram with vertical isomorphisms and horizontal quotient morphisms

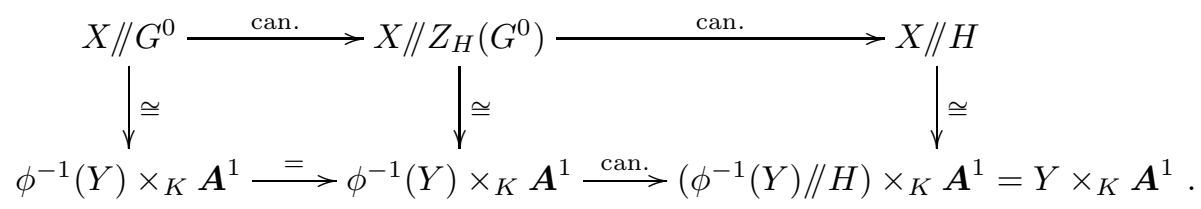

Since $\mathfrak{P} \cap \mathcal{O}(X)^{G^{0}}=\mathfrak{P}_{Y} \otimes_{K} \mathcal{O}(W)^{G^{0}}$ and $\mathfrak{P} \cap \mathcal{O}(X)^{H}=\left(\mathfrak{P}_{Y} \cap \mathcal{O}(Y)\right) \otimes_{K} \mathcal{O}(W)^{G^{0}}$, we see that

$$
\text { ht }\left(\mathfrak{P} \cap \mathcal{O}(\mathrm{X})^{\mathrm{H}}\right)=\mathrm{ht}\left(\mathfrak{P} \cap \mathcal{O}(\mathrm{X})^{\mathrm{G}^{0}}\right)=1
$$

and

$$
\mathrm{e}\left(\mathfrak{P} \cap \mathcal{O}(X)^{G^{0}}, \mathfrak{P} \cap \mathcal{O}(X)^{H}\right)=p .
$$

Put $M=\mathcal{O}(X)^{\mathcal{I}_{H}(\mathfrak{P})}$ and $S=M /\left(\mathfrak{P} \cap \mathcal{O}(X)^{\mathcal{I}_{H}(\mathfrak{P})}\right)$. The fact (6.3) implies $M=\mathcal{O}(X)$, $S=\mathcal{O}(X) / \mathfrak{P}$ and

$$
\mathrm{e}\left(\mathfrak{P}, \mathfrak{P} \cap \mathcal{O}(X)^{\mathcal{I}_{H}(\mathfrak{P})}\right)=1 .
$$

Then from Proposition 5.3 and (6.4) we infer that

$$
\begin{aligned}
\mathrm{e}(\mathfrak{P}, & \left.\mathfrak{P} \cap \mathcal{O}(X)^{\mathcal{I}_{H}(\mathfrak{P})}\right) \cdot \sharp_{p}\left(\left\langle\mathfrak{X}\left(G^{0}\right)^{M}\right\rangle /\left\langle\mathfrak{X}\left(G^{0}\right)^{S}\right\rangle\right) \\
& =\sharp_{p}\left(\left\langle\mathfrak{X}\left(G^{0}\right)^{M}\right\rangle /\left\langle\mathfrak{X}\left(G^{0}\right)^{S}\right\rangle\right) \\
& \leqq \mathrm{e}\left(\mathfrak{P}, \mathfrak{P} \cap \mathcal{O}(X)^{G^{0}}\right) \\
& <\mathrm{e}\left(\mathfrak{P}, \mathfrak{P} \cap \mathcal{O}(X)^{G^{0}}\right) \cdot p=\mathrm{e}\left(\mathfrak{P}, \mathfrak{P} \cap \mathcal{O}(X)^{H}\right) .
\end{aligned}
$$

This conflicts with the equality of (ii) for $X, H$ and $\mathfrak{P}$.

(i) $\Rightarrow$ (ii): We may suppose that $G=H=Z_{H}\left(G^{0}\right)$ and preserve notations in the circumstances as in (ii), i.e., let $(X, G)$ be an arbitrary effective regular action of $G$ on any affine Krull $K$-scheme $X=\operatorname{Spec}(R)$ such that $\mathcal{Q}(R)^{G^{0}}=\mathcal{Q}\left(R^{G^{0}}\right)$ and $\mathfrak{P} \in \operatorname{Ht}_{1}\left(R, R^{G}\right)$. For any finite subgroup $L$ of $G=Z_{G}\left(G^{0}\right)$, by Lemma 2.2 we see

$$
\mathcal{Q}(R)^{L \cdot G^{0}}=\mathcal{Q}\left(R^{L \cdot G^{0}}\right) .
$$

By (2.1) and Proposition 5.6, we have the equality

$$
\mathrm{e}_{p^{\prime}}\left(\mathfrak{P}, \mathfrak{P} \cap R^{G}\right)=\sharp_{p^{\prime}}\left(\left.\mathcal{I}_{G}(\mathfrak{P})\right|_{R}\right)=\mathrm{e}_{p^{\prime}}\left(\mathfrak{P}, \mathfrak{P} \cap R^{\mathcal{I}_{G}(\mathfrak{P})}\right)
$$

of $p^{\prime}$-parts of ramification indices. Consequently, in order to prove (ii), we suppose that $p>0$ and it suffices only to show the following equality

$$
\begin{aligned}
\mathrm{e}_{p}\left(\mathfrak{P}, \mathfrak{P} \cap R^{\mathcal{I}_{G}(\mathfrak{P})}\right) \cdot \sharp_{p}\left(\left\langle\mathfrak{X}\left(G^{0}\right)^{R^{\mathcal{I}_{G}(\mathfrak{P})}}\right\rangle /\left\langle\mathfrak{X}\left(G^{0}\right)^{\left.\left.R^{\mathcal{I}_{G}(\mathfrak{P})} / \mathfrak{P} \cap R^{\mathcal{I}_{G}(\mathfrak{P})}\right\rangle\right)}\right.\right. \\
=\mathrm{e}_{p}\left(\mathfrak{P}, \mathfrak{P} \cap R^{G}\right) .
\end{aligned}
$$


Put $\mathfrak{p}=\mathfrak{P} \cap R^{G^{0}}$ and $U=\left\langle\operatorname{Unip}\left(\mathcal{I}_{G}(\mathfrak{p})\right)\right\rangle$. By Proposition 4.1, the group $U$ is finite. Since $\mathcal{I}_{G}(\mathfrak{p}) \supseteq \mathcal{I}_{G}(\mathfrak{P})$ and $\mathcal{I}_{G}(\mathfrak{p}) \unrhd U$, by the definition of $U$ we see

$$
\mathcal{I}_{G}(\mathfrak{P}) \unrhd U \cap \mathcal{I}_{G}(\mathfrak{P})=\mathcal{I}_{U}(\mathfrak{P})
$$

and the factor group

$$
\mathcal{I}_{G}(\mathfrak{P}) / \mathcal{I}_{U}(\mathfrak{P})
$$

is of order which is not divisible by $p$. Thus

$$
\left(R^{\mathcal{I}_{U}(\mathfrak{P})} / \mathfrak{P} \cap R^{\mathcal{I}_{U}(\mathfrak{P})}\right)^{\mathcal{I}_{G}(\mathfrak{P}) / \mathcal{I}_{U}(\mathfrak{P})}=R^{\mathcal{I}_{G}(\mathfrak{P})} / \mathfrak{P} \cap R^{\mathcal{I}_{G}(\mathfrak{P})}
$$

and by Proposition 3.3 we see

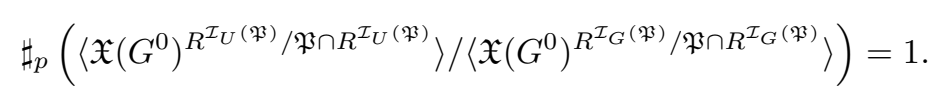

For the same reason,

$$
\sharp_{p}\left(\left\langle\mathfrak{X}\left(G^{0}\right)^{R^{\mathcal{I}_{U}(\mathfrak{P})}}\right\rangle /\left\langle\mathfrak{X}\left(G^{0}\right)^{R^{\mathcal{I}_{G}(\mathfrak{P})}}\right\rangle\right)=1 .
$$

By Proposition 3.6 we must have

$$
\sharp_{p}\left(\left\langle\mathfrak{X}\left(G^{0}\right)^{R^{\mathcal{I}_{U}(\mathfrak{p})} / \mathfrak{P} \cap R^{\mathcal{I}_{U}(\mathfrak{P})}}\right\rangle /\left\langle\mathfrak{X}\left(G^{0}\right)^{R^{U} / \mathfrak{P} \cap R^{U}}\right\rangle\right)=1
$$

and

$$
\begin{aligned}
& \sharp_{p}\left(\left\langle\mathfrak{X}\left(G^{0}\right)^{R^{U}}\right\rangle /\left\langle\mathfrak{X}\left(G^{0}\right)^{R^{U} / \mathfrak{P} \cap R^{U}}\right\rangle\right)
\end{aligned}
$$

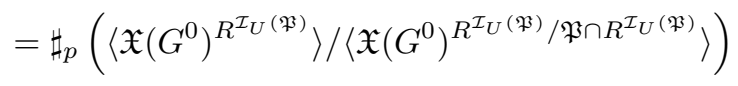

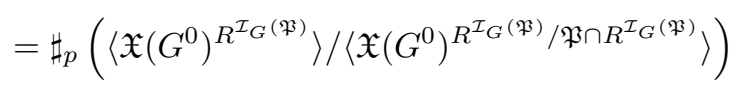

whose last equality follows from (6.6) and (6.7). On the other hand, applying Proposition 5.3 to the diagonal action $G^{0}$ on $R^{U}$ and on $R^{\mathcal{I}_{U}(\mathfrak{P})}$ respectively, we also must have

$$
\begin{aligned}
& \mathrm{e}\left(\mathfrak{P} \cap R^{U}, \mathfrak{P} \cap R^{U \cdot G^{0}}\right)=\sharp\left(\left\langle\mathfrak{X}\left(G^{0}\right)^{R^{U}}\right\rangle /\left\langle\mathfrak{X}\left(G^{0}\right)^{R^{U}} / \mathfrak{P} \cap R^{U}\right\rangle\right) \\
& \mathrm{e}\left(\mathfrak{P} \cap R^{\mathcal{I}_{U}(\mathfrak{P})}, \mathfrak{P} \cap R^{\mathcal{I}_{U}(\mathfrak{P}) \cdot G^{0}}\right)=\sharp\left(\left\langle\mathfrak{X}\left(G^{0}\right)^{R^{\mathcal{I}_{U}(\mathfrak{P})}}\right\rangle /\left\langle\mathfrak{X}\left(G^{0}\right)^{\left.\left.R^{\mathcal{I}_{U}(\mathfrak{P})} / \mathfrak{P} \cap R^{\mathcal{I}_{U}(\mathfrak{P})}\right\rangle\right)}\right.\right.
\end{aligned}
$$

These equalities and (6.8) imply

$$
\mathrm{e}_{p}\left(\mathfrak{P} \cap R^{U}, \mathfrak{P} \cap R^{U \cdot G^{0}}\right)=\mathrm{e}_{p}\left(\mathfrak{P} \cap R^{\mathcal{I}_{U}(\mathfrak{P})}, \mathfrak{P} \cap R^{\mathcal{I}_{U}(\mathfrak{P}) \cdot G^{0}}\right) .
$$

By (iii) of Proposition 4.3 ,

$$
\mathrm{e}_{p}\left(\mathfrak{P} \cap R^{U \cdot G^{0}}, \mathfrak{P} \cap R^{G}\right)\left(=\mathrm{e}_{p}\left(\mathfrak{p} \cap R^{U \cdot G^{0}}, \mathfrak{p} \cap R^{G}\right)\right)=1 .
$$

Clearly e $\left(\mathfrak{P}, \mathfrak{P} \cap R^{\mathcal{I}_{U}(\mathfrak{P})}\right)=\mathrm{e}\left(\mathfrak{P}, \mathfrak{P} \cap R^{U}\right)$. Consider the action $\mathcal{I}_{G}(\mathfrak{P}) / \mathcal{I}_{U}(\mathfrak{P})$ on $R^{\mathcal{I}_{U}(\mathfrak{P})}$. Because

$$
\mathcal{I}_{\mathcal{I}_{G}(\mathfrak{P}) / \mathcal{I}_{U}(\mathfrak{P})}\left(\mathfrak{P} \cap R^{\mathcal{I}_{U}(\mathfrak{P})}\right)=\mathcal{I}_{G}(\mathfrak{P}) / \mathcal{I}_{U}(\mathfrak{P}),
$$

by ramification theory we see that the ramification index

$$
\mathrm{e}\left(\mathfrak{P} \cap R^{\mathcal{I}_{U}(\mathfrak{P})}, \mathfrak{P} \cap R^{\mathcal{I}_{G}(\mathfrak{P})}\right)
$$


is a divisor of $\sharp\left(\mathcal{I}_{G}(\mathfrak{P}) / \mathcal{I}_{U}(\mathfrak{P})\right)$, which is not divisible by $p$. Consequently by (6.8), (6.9), (6.10), 6.11) and 6.12) we must have

$$
\begin{aligned}
\mathrm{e}_{p}\left(\mathfrak{P}, \mathfrak{P} \cap R^{G}\right) & =\mathrm{e}_{p}\left(\mathfrak{P}, \mathfrak{P} \cap R^{U}\right) \cdot \mathrm{e}_{p}\left(\mathfrak{P} \cap R^{U}, \mathfrak{P} \cap R^{U \cdot G^{0}}\right) \\
& =\mathrm{e}_{p}\left(\mathfrak{P}, \mathfrak{P} \cap R^{\mathcal{I}_{U}(\mathfrak{P})}\right) \cdot \mathrm{e}_{p}\left(\mathfrak{P} \cap R^{\mathcal{I}_{U}(\mathfrak{P})}, \mathfrak{P} \cap R^{\mathcal{I}_{U}(\mathfrak{P}) \cdot G^{0}}\right) \\
& =\mathrm{e}_{p}\left(\mathfrak{P}, \mathfrak{P} \cap R^{\mathcal{I}_{U}(\mathfrak{P})}\right) \cdot \sharp_{p}\left(\left\langle\mathfrak{X}\left(G^{0}\right)^{R^{\mathcal{I}_{U}(\mathfrak{P})}}\right\rangle /\left\langle\mathfrak{X}\left(G^{0}\right)^{\left.\left.R^{\mathcal{I}_{U}(\mathfrak{P})} / \mathfrak{P} \cap R^{\mathcal{I}_{U}(\mathfrak{P})}\right\rangle\right)}\right.\right. \\
& =\mathrm{e}_{p}\left(\mathfrak{P}, \mathfrak{P} \cap R^{\mathcal{I}_{G}(\mathfrak{P})}\right) \cdot \sharp_{p}\left(\left\langle\mathfrak{X}\left(G^{0}\right)^{R^{\mathcal{I}_{G}(\mathfrak{P})}}\right\rangle /\left\langle\mathfrak{X}\left(G^{0}\right)^{\left.\left.R^{\mathcal{I}_{G}(\mathfrak{P})} / \mathfrak{P} \cap R^{\mathcal{I}_{G}(\mathfrak{P})}\right\rangle\right)}\right.\right.
\end{aligned}
$$

which shows 6.5 required as above. Thus the proof is completed.

Example 6.2 Case " $G^{0}$ is a simple algebraic group" : Let $k$ be an odd natural number $>1$ such that $k$ is not divisible by $p$ if $p>0$ and $\zeta_{k}$ be a fixed primitive $k$-th root of $1 \in K$. Define the subgroup

$$
G:=\left\langle S L_{2}(K),\left[\begin{array}{cc}
\zeta_{k} & 0 \\
0 & \zeta_{k}
\end{array}\right]\right\rangle \subseteq G L_{2}(K)
$$

which acts naturally on the $K$-vector space $K X_{1} \oplus K X_{2}$. The group $G$ is an affine algebraic group with $G^{0}=S L_{2}(K)$ and $G=Z_{G}\left(G^{0}\right) \cdot G^{0}$. Let $R$ be a polynomial ring $K\left[Y_{1}, Y_{2}, Y_{3}\right]$ over $K$ with three variables. Identifying

$$
Y_{1}=X_{1}^{2}, Y_{2}=X_{1} X_{2} \text { and } Y_{3}=X_{2}^{2}
$$

the group $G$ acts on $R$ induced from the representation on the homogeneous part of $K\left[X_{1}, X_{2}\right]$ of degree 2 . Then there is a homogeneous polynomial $f$ of $R$ of degree 2 such that $R^{G^{0}}=K[f]$. Clearly $f$ is a prime element of $R$ and $\mathfrak{P}:=R f \in \operatorname{Ht}_{1}\left(R, R^{G}\right)$. For any finite subgroup $\Gamma$ of $G$, we have

$$
\mathrm{e}\left(\mathfrak{P}, \mathfrak{P} \cap R^{\Gamma}\right)=\mathrm{e}\left(\mathfrak{P}, \mathfrak{P} \cap R^{\mathcal{I}_{\Gamma}(\mathfrak{P})}\right)=1,
$$

because

$$
\mathcal{I}_{\Gamma}(\mathfrak{P}) \subseteq \mathfrak{R}(R, G) \subseteq Z_{G}\left(G^{0}\right) \subseteq Z\left(G L_{2}(K)\right) \quad(c f .[12])
$$

However

$$
\mathrm{e}\left(\mathfrak{P}, \mathfrak{P} \cap R^{G}\right)=k>\mathrm{e}\left(\mathfrak{P}, \mathfrak{P} \cap R^{\Gamma}\right),
$$

which shows that Problem 1.1 is not affirmative in this case.

\section{References}

[1] N. Bourbaki, Groupes et Algèbres de Lie : Chapitres 4 à 6 (Éléments de Mathématique) Springer-Verlag, Berlin-Heidelberg-New York, 2006.

[2] N. Bourbaki, Commutative Algebra : Chapters 1-7 (Elements of Mathematics) SpringerVerlag, Berlin-Heidelberg-New York, 1989.

[3] Robert M. Fossum, The Divisor Class Groups of a Krull Domains, Springer-Verlag, Berlin-Heidelberg-New York, 1973.

[4] A. Grothendieck; Michèle, Raynaud, Revêtements Étales et Groupe Fondamental (SGA1), Seminaire de geometrie algebrique du Bois-Marie 1960-61, Documents Mathématiques (Paris) 3, Société Mathématique de France, 2003.

[5] Andy Magid, Finite generation of class groups of rings of invariants, Proc. of Amer. Math. Soc. 60 (1976), 45-48. 
[6] Hideyuki Matsumura, Commutative Ring Theory, Cambridge Univ. Press, Cambridge, 1986.

[7] Masayoshi Nagata, Local Rings, Robert E. Krieger Publ. Co., New York, 1975. Math. Zeit 182 (1983), 1-15.

[8] H. Nakajima, Relative invariants of finite groups, J. Algbera 79 (1982), 218-234.

[9] H. Nakajima, Algebraic tori admitting finite central coregular extensions, Proc. Japan Acad. Ser. A Math. Sci. 68 (1992), 273-278.

[10] H. Nakajima, Reduced ramification indices of quotient morphisms under torus actions, J. Algebra 242 (2001), 536-549.

[11] H. Nakajima, Reduced class groups grafting relative invariants Advances in Math. 227 (2011), 920-944.

[12] H. Nakajima, Reductivities and finiteness of pseudo-reflections of algebraic groups and homogeneous fiber bundles J. Pure and Appl. Algebra 217 (2013), 1548-1562.

[13] Vladimir L. Popov; Tonny A. Springer, Algebraic Geometry IV, Encyclopaedia of Mathematical Sciences 55, Springer-Verlag, Berlin-Heidelberg-New York, 1994.

[14] D. I. Panyushev, On semisimple groups admitting a finite coregular extension, Funct. Analysis and Its Appl., 27 (1993), 216-217.

[15] R. Pries; K. Stevenson, A survey of Galois theory of curves in characterristic $p$, Fields Inst. Commun. 60 (2011), 169-191.

[16] Richard P. Stanley, Relative invariants of finite groups generated by pseudo-reflections, J. Algbera 49 (1977), 134-148.

[17] Oscar Zariski; Pierre Samuel, Commutative Algebras I Graduate Texts in Math. 28 Springer-Verlag, Berlin/Heidelberg/New York, 1975. 